\title{
Antinociceptive Effects of Alpinia katsumadai via Cyclooxygenase-2 Inhibition
}

\author{
Jin Kyu ChоI ${ }^{1}$, Kwang-Mi Kıм ${ }^{1}$, Myeong-Hoon Yеом ${ }^{1}$, Hee Yeong $\mathrm{CHO}^{2}$, Hye Ja LeE ${ }^{3}$, Mi Kyung ParK ${ }^{3}$, \\ Kyung Chae JeONG ${ }^{4}$, Byung II LeE ${ }^{4}$, Minsoo $\mathrm{NoH}^{1}$, and Chang Hoon LeE ${ }^{3, *}$ \\ ${ }^{1} R$ \& D Center, AmorePacific Corporation, Yongin 449-729, ${ }^{2}$ Korea Research Institute of Chemical Technology, Daejeon 305-600, \\ ${ }^{3}$ Department of Life Science, Dongguk University, Seoul 100-715, ${ }^{4}$ National Cancer Center, Goyang 416-769, Republic of Korea
}

(Received January 21, 2010; Revised February 24, 2010; Accepted March 24, 2010)

\begin{abstract}
Alpinia katsumadai has been widely used in traditional Chinese and Korean medicine to treat a variety of conditions including emesis and gastric disorders such as gastric pain and distended abdomen. To investigate the antinociceptive potential and mechanism of $A$. katsumadai, ethanolic extracts of $A$. katsumadai were assayed on cyclooxygenase-2 and evaluated for analgesic activity based on phenylbenzoquinone (PBQ)-induced writhing and carrageenan-induced hyperalgesia tests. A. katsumadai extracts inhibited the cyclooxygenase-2 enzyme activity in a dose-dependent fashion at an $\mathrm{IC}_{50}$ value of $0.044 \mu \mathrm{g} / \mathrm{ml}$. A. katsumadai extract (30-300 mg/kg, orally (p.o.) administered) significantly inhibited PBQ-induced writhing. This inhibition was judged not to be a false positive because a Rota-rod test revealed no difference in muscular coordination when compared to the controls. With regard to the carrageenan-induced hyperalgesia, A. katsumadai extract (30-300 mg/kg, p.o.) produced a significant, dose-dependent increase in the withdrawal response latencies. Naloxone did not reverse the analgesic effect of $A$. katsumadai extract in the carrageenan-induced hyperalgesia. Taken together, these results suggest that the antinociceptive activity of A. katsumadai is not related to the opioid receptor. A. katsumadai extract has remarkable, non-opioidreceptor-mediated analgesic effects on $\mathrm{PBQ}$-induced writhing and carrageenan-induced hyperalgesia that occur via cyclooxygenase-2 inhibition.
\end{abstract}

Keywords: Alpinia katsumadai, Analgesic, Anti-inflammatory, Antinociceptive, Phenylbenzoquinone

\section{INTRODUCTION}

The plant used in this study, Alpinia katsumadai, was selected based on the use of its seeds in traditional Chinese and Korean medicine (Ching, 1978; Kim et al., 2000). A. katsumadai has been widely used in traditional Chinese and Korean medicine to treat a variety of conditions including emesis and gastric disorders such as gastric pain and distended abdomen. Alpinia katsumadai is often used with other herbs such as cinnamon bark and ginger (Dong et al., 1992). The extract from A. katsumadai seeds is known to have significant antioxidant activity (Lee et al., 2003), antiemetic activity (Yang et al., 1999) and to inhibit prostaglandin production in LPS-stimulated mouse peritoneal macrophages (Noh et al., 1998).

${ }^{*}$ Corresponding author

Tel: +82-2-2260-8950 Fax: +82-2-2260-8905

E-mail: uatheone@gmail.com
In addition, we recently reported that $A$. katsumadai has anti-pruritic activities in a thromboxane $\mathrm{A}_{2}$-involved mouse model (Choi et al., 2009). The traditional use of $A$. katsumadai for the treatment of gastric pain and distended abdomen suggest that the antinociceptive and anti-inflammatory activity of $A$. katsumadai may play a role in the use of this plant. However, very little is known about the antinociceptive activities of $A$. katsumadai even though it is already known that some other Alpinia species have antinocicpetive effects. Therefore, in the current investigation, we assessed the analgesic potential of $A$. katsumadai extract in animals as well as the related mechanism of the antinociceptive effects.

\section{MATERIALS AND METHODS}

\section{Chemicals and animals}

Indomethacin, carrageenan and phenylbenzoquinone 
(PBQ) were purchased from Sigma (St. Louis, USA).

All of the mice and rats used in the present study were purchased from Samtako Korea and maintained under SPF conditions until required. The animals were housed under standard conditions $\left(22 \pm 2^{\circ} \mathrm{C}, 50 \pm 10 \%\right.$ humidity and a $12 \mathrm{~h}$ light-dark cycle) and maintained with free access to water and a regular diet.

Male Sprague-Dawley rats (6-7 weeks) and male ICR mice (5-6 weeks) were used in the experiments, and all experiments were conducted under the Korea Association for Laboratory Animal Science's guidelines for the care and use of experimental animals.

\section{Preparation of $\boldsymbol{A}$. katsumadai extract}

Dried seeds of $A$. katsumadai were purchased at the Kyungdong Oriental Drug Store (Suwon, Korea) in 2007. The materials obtained were authenticated by Professor Sun Yeou Kim of the Department of Medical Science, Graduate School of East West Medical Science, Kyung Hee University, Korea. A voucher specimen (PRI99-121) has been deposited in the Natural Resource Deposit, Amorepacific R\&D Center. A. katsumadai $(200 \mathrm{~g})$ was extracted at $50^{\circ} \mathrm{C}$ in $95 \%$ ethanol for $15 \mathrm{~h}$, after which the extract was filtered and the filtrate was concentrated in a vacuum rotary evaporator under low pressure.

\section{Fingerprint of $\boldsymbol{A}$. katsumadai extracts by gas chromato- graphy-mass spectrometry (GC-MS)}

An Agilent 6890 gas chromatograph coupled with a JEOL GC-Mate 2 mass spectrometer was used to establish the fingerprint of $A$. katsumadai extracts. The separation was performed on a HP-5MS column $(0.25 \mathrm{~mm}$ i.d. $X$ $30 \mathrm{~m}, 0.32 \mu \mathrm{m}$ coating thickness). The temperature of the column was programmed to increase from 50 to $300^{\circ} \mathrm{C}$ at $10^{\circ} \mathrm{C} /$ min during analysis, while the injector temperature and the detector temperature were maintained at $280^{\circ} \mathrm{C}$. Helium was applied as the carrier gas at a constant flow rate of $1.0 \mathrm{ml} / \mathrm{min}$. The mass spectrometer was operated at $70 \mathrm{eV}$ and a scan range of 5-500 amu. Some of the compounds were identified from the recorded mass spectra by comparison with the mass spectra from the NIST and Wiley libraries.

\section{Cyclooxygenase-2 enzyme assay}

The cyclooxygenase-2 activity was determined by a spectrophotometric assay conducted using recombinant ovine COX-2 enzymes (Cayman Chemicals, Ann Arbor, MI) (Ouellet et al., 2001). Briefly, 50 nM COX-2 in 100 mM Tris buffer $(\mathrm{pH} 8.0)$ and $0.5 \mathrm{mM}$ heme were added to the appropriate wells. The reaction was then initiated with 100
$\mathrm{mM}$ arachidonic acid and $10 \mathrm{mM}$ TMPD $\left(\mathrm{N}, \mathrm{N}, \mathrm{N}^{\prime}, \mathrm{N}^{\prime}\right.$-tetrametyl-p-phenylenediamine) after incubation with the extracts of $A$. katsumadai for 5 min under the indicated concentrations. After $5 \mathrm{~min}$ of incubation at room temperature absorbance at $590 \mathrm{~nm}$ was read. Indomethacin was used as a reference compound.

\section{Phenylbenzoquinone (PBQ)-induced writhing in mice}

The writhing test was conducted according to the modified method described by Siegmund et al. (1957). Acute pain was induced by intraperitoneal injection of $0.2 \mathrm{ml}$ of $0.02 \%$ PBQ 54 min after p.o. administration of the A. katsumadai extract or other compounds. Six min after the PBQ injection, the total number of writhes was counted for $6 \mathrm{~min}$. The control animals received an appropriate volume of dosing vehicle $(80 \%$ saline, $10 \%$ ethanol and $10 \%$ Tween 80). Indomethacin was used as a positive control.

\section{Carrageenan-induced hyperalgesia test in rats}

The carrageenan-induced hyperalgesia test was conducted according to the modified method described by Randall and Selitto (1957) (Siegmund et al., 1957). The nociceptive pressure threshold was determined for the left hindpaw (Analgesymeter, Ugo Basile, Milan) using a wedge-shaped mechanical probe to apply graded pressure stimuli to the paw. The cut-off threshold was set at $250 \mathrm{~g}$. The end point was taken as paw withdrawal, vocalization or overt struggling. The nociceptive pressure thresholds were determined in the absence of inflammation. The rats were divided into groups of six according to their nociceptive pressure thresholds, after which carrageenan $(0.1 \mathrm{ml}, 1 \%)$ was injected into the plantar surface of the left hindpaw. The rats received vehicle or compounds $2 \mathrm{~h}$ after carrageenan injection and were evaluated for paw hyperalgesia 0,1 , and $2 \mathrm{~h}$ after compounds administration. To investigate the involvement of the opioid receptor, the rats were injected with $5 \mathrm{mg} / \mathrm{kg}$ of naloxone hydrochloride (s.c.) and $A$. katsumadai extract (p.o.) simultaneously.

\section{Carrageenan-induced hind paw edema test in rats}

The carrageenan-induced hind paw edema test was conducted according to the modified method described by Winter et al. (1962). Briefly, the rats were divided randomly into groups of five, after which they were injected subcutaneously through the plantar surface of the hind paw with $0.05 \mathrm{ml}$ of $1 \%$ carrageenan. Different doses of plant extract were injected p.o. $1 \mathrm{~h}$ before the administration of carrageenan. Indomethacin was used as a positive control. Rat paw edema was assessed using a plethysmometer (Ugobasile 7150) before and $3 \mathrm{~h}$ after the carra- 
geenan injection. The difference in paw volume determined before and after the carrageenan injection was taken to indicate the severity of edema.

\section{Rota-rod test in mice on muscular coordination}

The rota-rod (Ugo Basile, model-7600) consisted of a bar with a diameter of $2.5 \mathrm{~cm}$ subdivided into five compartments by disks $25 \mathrm{~cm}$ in diameter. The bar rotated at a constant speed of 30 revolutions per minute. Mice were tested at $1 \mathrm{~h}$ after administration of the extracts. This test requires a high degree of sensory motor coordination and is therefore used to test more subtle neurological deficits than those determined by loss of the righting reflex. All mice utilized were initially tested and shown to be able to pass the rota-rod test prior to drug administration and subsequent evaluation.

\section{Statistical data analysis}

All data are presented as means \pm S.E. Statistical significance $(p<0.05)$ was determined by one-way analysis of variance (ANOVA) followed by Dunnett's test. $p<0.05$ relative to the control group was considered to be significant.

\section{RESULTS}

\section{The fingerprint of $\boldsymbol{A}$. katsumadai extracts established by gas chromatography-mass spectrometry (GC-MS)}

The fingerprint of $A$. katsumadai extracts was obtained by GC-MS (Fig. 1). Based on the chromatogram of the extracts, four main component peaks were detected: eucalyptol (retention time: $7.00 \mathrm{~min}$ ), hexadecanoic acid (retention time: $19.14 \mathrm{~min}$ ), oleic acid (retention time: 20.57 $\mathrm{min}$ ) and 4,6-heptadien-3-one, 1,7-diphenyl (retention time: $24.09 \mathrm{~min}$ )

\section{Effects of $\boldsymbol{A}$. katsumadai on cyclooxygenase-2 enzyme activity}

We reported that the extracts of $A$. katsumadai suppressed the production of $\mathrm{PGE}_{2}$ in LPS-treated macrophages (Noh et al., 1998). Recently, we showed that the suppression of $\mathrm{PGE}_{2}$ production was due to reduced expression of COX-2 (Choi et al., 2009). Therefore, this study was conducted to examine the direct effects of $A$. katsumadai extracts on COX-2 enzyme. The results revealed that $A$. katsumadai extracts significantly suppressed COX2 enzyme activity (Table I) and that the $\mathrm{IC}_{50}$ of the extract against COX-2 was $0.044 \mu \mathrm{g} / \mathrm{ml}$.

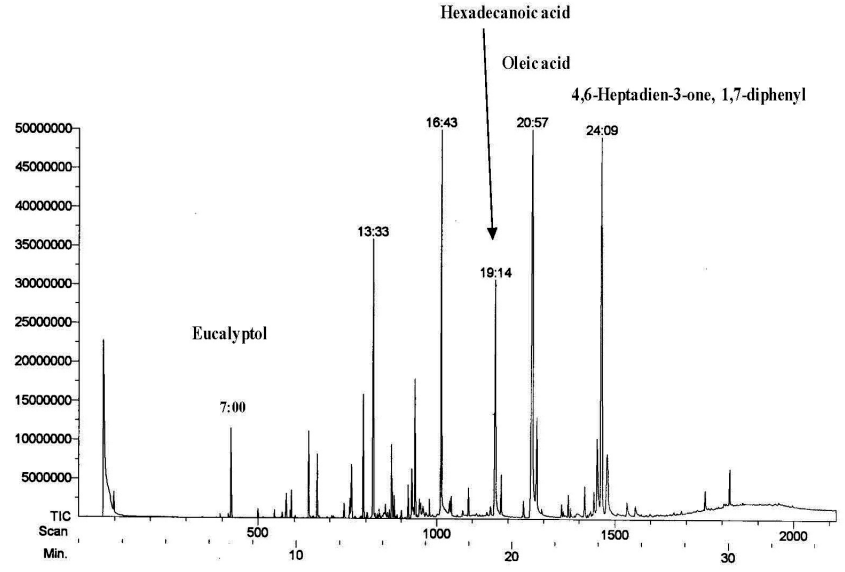

Fig. 1. The fingerprint of the $A$. katsumadai extracts obtained by GC-MS showing several compounds and four main compounds were identified: Eucalyptol (retention time 1/4 $7.00 \mathrm{~min}$ ), Hexadecanoic acid (retention time $1 / 419.14 \mathrm{~min}$ ), Oleic acid (retention time $1 / 420.57 \mathrm{~min}$ ) and 4,6-Heptadien-3-one, 1,7-diphenyl (retention time $1 / 424.09 \mathrm{~min}$ ).

Table I. COX-2 inhibitory effect of $A$. katsumadai

\begin{tabular}{lc}
\hline A. katsumadai $(\mu \mathrm{g} / \mathrm{ml})$ & $\%$ inhibition $( \pm$ S.D. $)$ \\
\hline 0.003 & $-5.26 \pm 1.55$ \\
0.006 & $0.533 \pm 2.62$ \\
0.013 & $9.30 \pm 6.36$ \\
0.025 & $29.9 \pm 1.82$ \\
0.05 & $50.1 \pm 2.89$ \\
0.1 & $60.0 \pm 3.02$ \\
Indomethacin $^{a}$ & $68.4 \pm 5.45$
\end{tabular}

${ }^{\mathrm{a}}$ Indomethacin was used as a reference compound $(10 \mu \mathrm{M})$.

\section{Effects of $A$. katsumadai on phenylbenzoquinone (PBQ)- induced writhing test in mice}

To assess the in vivo analgesic activity of $A$. katsumadai extracts, they were first evaluated in mice using the phenylbenzoquinone (PBQ)-induced writhing test. Intraperitoneal administration of $\mathrm{PBQ}$ induced $22 \pm 2(\mathrm{n}=6)$ writhes in control rats during the 6 min observation period. However the animals that received $A$. katsumadai at doses of 30 , 100 and $300 \mathrm{mg} / \mathrm{kg}$ body wt. writhed $16 \pm 1(\mathrm{n}=6), 15 \pm 2$ $(n=6)$, and $11 \pm 1(n=6)$ times, respectively (Fig. 2A). For comparison, a group of animals administered $1 \mathrm{mg} / \mathrm{kg}$ body wt. indomethacin 30 min prior to noxious stimulation was evaluated and the number of writhes was found to be $13 \pm 3(n=6)$. Only treatment with $A$. katsumadai at 300 $\mathrm{mg} / \mathrm{kg}$ body wt. induced a significant reduction in the writhing response $(p<0.05$, ANOVA, Dunnett's test). 


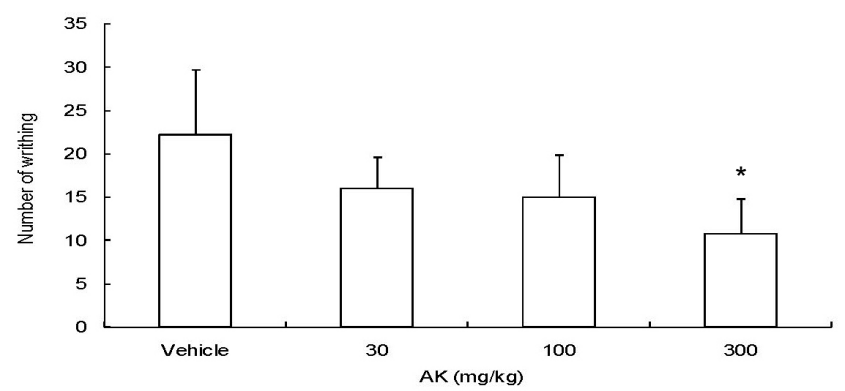

B.

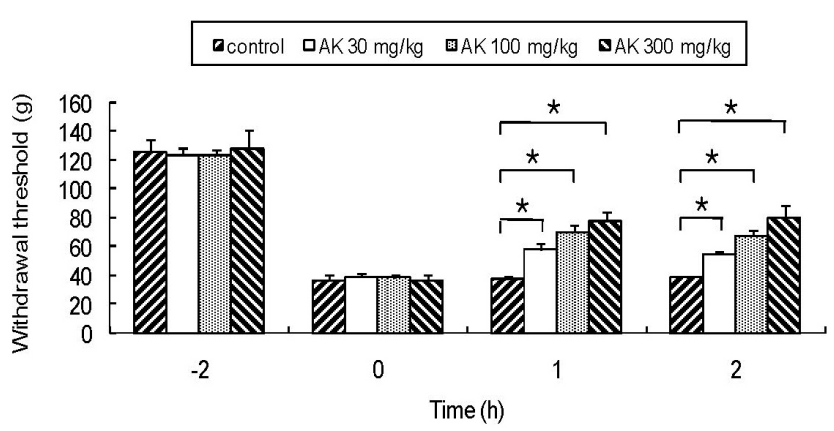

Fig. 2. (A) Effect of $A$. katsumadai (AK) plant extract and indomethacin on $P B Q$ induced writhing in mice $(n=6$; mean \pm $\mathrm{SE})$. As compared with control: ${ }^{*} p<0.05$, ANOVA ( $p=0.011$, $F=4.87$ ), Dunnett's test. (B) Effect of different doses of the extract of $A$. katsumadai on the hind paw withdrawal threshold of rats $(n=5$; carrageenan-induced hyperalgesia test, mean \pm $\mathrm{SE})$. As compared with control of each indicated time point: * $p$ $<0.05$, ANOVA (1 h: $p=0.000, \mathrm{~F}=16.21,2 \mathrm{~h}: p=0.000, \mathrm{~F}=15.67)$, Dunnett's test.

\section{Effects of $\boldsymbol{A}$. katsumadai on carrageenan-induced hyper- algesia in rats}

To determine if $A$. katsumadai had an analgesic effect, the rats were evaluated by means of a carrageenan-induced mechanical hyperalgesia study. The results revealed significant prolongation of the paw withdrawal threshold in rats treated with $A$. katsumadai extract at 1 and $2 \mathrm{~h}$ post-treatment (Fig. 2B). To determine if $A$. katsumadai produced an analgesic activity independent of the endogenous opiate system, the interaction between $A$. katsumadai and naloxone was evaluated. Furthermore, the results revealed that subcutaneous administration of naloxone did not significantly reduce the paw withdrawal threshold increased by the $300 \mathrm{mg} / \mathrm{kg}$ A. katsumadai extract (Fig. 3A).
A.

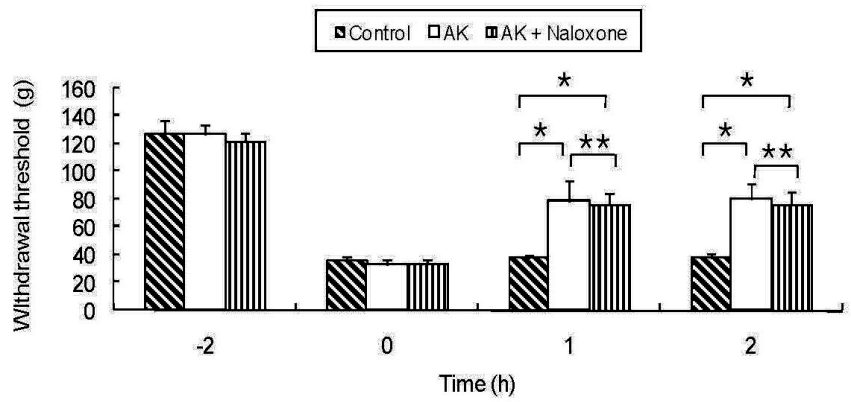

B.

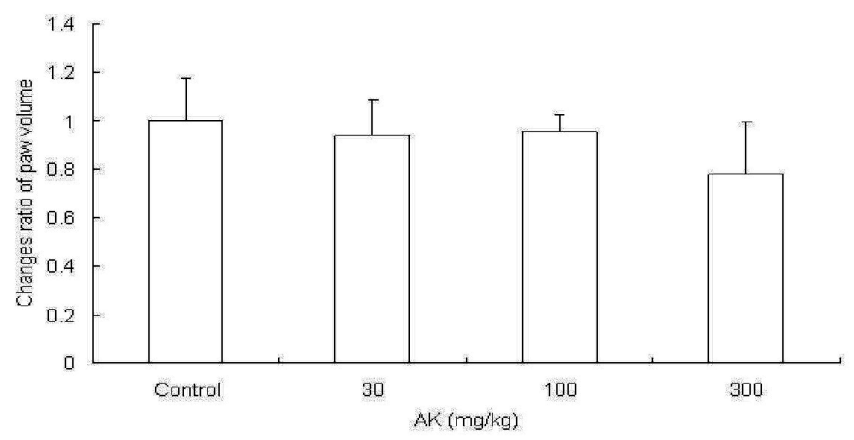

Fig. 3. (A) Effect of naloxone on the reaction time of rats induced by $300 \mathrm{mg} / \mathrm{kg} \mathrm{A}$. katsumadai (AK) extract ( $\mathrm{n}=5-6$; carrageenan-induced hyperalgesia test, mean $\pm \mathrm{SE}$ ). As compared with control of each indicated time point: ${ }^{*} p<0.05$, ANOVA ( $1 \mathrm{~h}$ : $p=0.007, \mathrm{~F}=7.48,2 \mathrm{~h}: p=0.003, \mathrm{~F}=9.16)$, Dunnett's test, ${ }^{* *}$ No differences. (B) Effect of $A$. katsumadai plant extract on carrageenan induced paw edema in rats $(n=5$, mean $\pm S E)$. ANOVA $(p=0.195, \mathrm{~F}=1.76)$.

\section{Effects of $\boldsymbol{A}$. katsumadai on carrageenan-induced hind paw edema test in rats}

Measurement of footpad edema is a convenient method of assessing inflammatory response. A. katsumadai was assessed for acute anti-inflammatory action by examining its ability to reduce carrageenan-induced paw swelling. Animals injected with carrageenan and treated with vehicle displayed significant edema in the ipsilateral paw $3 \mathrm{~h}$ post-carrageenan treatment ( $p<0.05$ vs pre-carrageenan values; data not shown). Furthermore, p.o. administration of $A$. katsumadai extract ( $30-100 \mathrm{mg} / \mathrm{kg}$ ) did not cause significant inhibition of the edema induced by sub-plantar carrageenan injection. A high dose $(300 \mathrm{mg} / \mathrm{kg})$ of $A$. katsumadai induced a slight suppression of the edema, but this effect was not statistically significant ( $p>0.05$ vs control, ANOVA, Dunnett test; Fig. 3B). 
Table II. Treatment with chlorpromazine with the extract of $A$. katsumadai (p.o.) in rota-rod tests in mice

\begin{tabular}{lcc}
\hline \multicolumn{1}{c}{ Treatments } & Dose $\left(\mathrm{mg} \mathrm{kg}^{-1}\right)$ & Number of fallen mice \\
\hline Vehicle $^{\mathrm{b}}$ & 0 & $0 / 5$ \\
Alpinia katsumadai & 300 & $0 / 5$ \\
Chlopromazine & 10 & $5 / 5$ \\
\hline
\end{tabular}

${ }^{a}$ Within 2 min, ${ }^{b}$ Vehicle: ethanol-tween 80-saline (1:1:8).

\section{Effect of $\boldsymbol{A}$. katsumadai on rota-rod test in mice}

To exclude the possible non-specific action of the extract, animals were subjected to the rota-rod test to measure motor performance according to the method described by Duham and Miya, with minor modification (Dunham and Miya, 1957).

High-dose oral administration of $A$. katsumadai extracts $(300 \mathrm{mg} / \mathrm{kg}$ ) did not cause the latency to decrease significantly in the rota-rod test $(p>0.05)$. However, the reference drug, chlorpromazine $(10 \mathrm{mg} / \mathrm{kg})$, reduced the latency (Table II). Taken together, these findings indicate that the effects of $A$. katsumadai extracts on PBQ-induced writhing were not caused by impaired motor functions.

\section{DISCUSSION}

Analgesic therapeutic alternatives have been found to be only partially effective, and their application is commonly associated with severe side-effects that hinder their continuous use (Mendell and Sahenk, 2003). The interest in identifying new alternatives to treat inflammatory pain has increased greatly in recent years (Calixto et al., 2004), and naturally occurring plants seem to represent good choices for this purpose.

As shown in Fig. 1, the fingerprint of $A$. katsumadai showed the existence of eucalyptol (retention time: 7.00 $\mathrm{min}$ ), hexadecanoic acid (retention time: $19.14 \mathrm{~min}$ ), oleic acid (retention time: $20.57 \mathrm{~min}$ ) and 4,6-heptadien-3-one, 1,7-diphenyl (retention time: $24.09 \mathrm{~min}$ ), which is consistent with the results of a previous study conducted to evaluate A. katsumadai (Kuroyanagi, 1983; Yang et al., 1999).

As shown in Table I, A. katsumadai extracts showed strong inhibitory effects against COX-2 enzyme activity, as indicated by an $\mathrm{IC}_{50}$ of $0.044 \mu \mathrm{g} / \mathrm{ml}$. These findings were somewhat surprising, and it is not clear which components of $A$. katsumadai are responsible for this highly potent inhibition. Diarylheptanoids from other Alpinia species have showed inhibitory effects against COX-2 enzyme and it has previously been reported that $A$. katsumadai contained diarylheptanoids, which was also confirmed by our fingerprint experiment (Yang et al., 1999) (Fig. 1). Taken together, these findings indicate that the diarylheptanoids from $A$. katsumadai may be potential COX-2 inhibitors.

The results of the present study also demonstrated that extracts of the seeds of $A$. katsumadai have antinociceptive activity as indicated by the PBQ-induced writhing and carrageenan-induced hyperalgesia test (Fig. 2). The PBQ-induced writhing test, which is generally used to assess the antinociceptive activity in mice, is known to be devoid of limitations due to interference drugs that inhibit writhing other than analgesics, such as antihistaminics, sympathomimetics, and parasympathomimetic-like central nervous system stimulants and adrenergic blockers (Bjorkman et al., 1994). Accordingly, this writhing test points out analgesic activity of tested material acting either through a peripheral or a central mechanism of action (Hendershot and Forsaith, 1959). Therefore, some sedatives possess antinociceptive activity (Rang et al., 1978). However, the antinociceptive activity of the $A$. katsumadai extract was found to be genuine in the present study. Specifically, the rota-rod test revealed no difference in muscular coordination when compared with the control, but the reference drug, chlorpromazine $(10 \mathrm{mg} / \mathrm{kg})$, reduced the latency (Table II).

There is compelling evidence linking bradykinin, prostaglandin $E_{2}$, leukotriene $B_{4}, P A F$, interleukin 1, 5-hydroxytryptamine and histamine to the pathophysiological processes that accompany tissue damage and inflammation, especially the production of pain and hyperalgesia (Steen et al. 1996; Smith et al., 1998). Carrageenan-induced hyperalgesia might be provoked via a central or peripheral action of mechanism. The fact that naloxone, which is a classical morphine receptor antagonist (Gracioso et al., 1998), did not block the antinociception induced by the $A$. katsumadai extract suggests that the antinociception was not mediated through opioid receptors or the release of endogenous opioid substances (Fig. 3). Thus $A$. katsumadai extract might not produce side effects of opioid tolerance such as physical dependence and withdrawal phenomena.

It has already been reported that two species of Alpinia, leaves of $A$. zerumbet (de Araujo et al., 2005) and rhizomes of A. calcarata (Arambewela et al., 2004), exhibit analgesic activities via the opioid receptor. It is quite interesting that the opioid receptor was not involved in the antinociceptive activity of $A$. katsumadai (Fig. 3B). This mechanistic difference is due to the varying compositions of compounds found in the different parts of $A$. katsumadai. For example, the composition of the compounds of the seeds of $A$. katsumadai is different from that of other parts such as the leaves of $A$. zerumbet and rhizomes of $A$. 
calcarata. The mechanism underlying this analgesic effect of the seeds of $A$. katsumadai remains unknown, but appears to be related to peripheral nociceptive signaling since the classical morphine receptor antagonist naloxone did not block the antinociception induced by the $A$. katsumadai extract.

A. katsumadai has long been used to treat gastric pain and distended abdomen, but it has not been determined what component and biological activity of $A$. katsumadai are responsible for its analgesic effects. It is possible that the antinociceptive effect of $A$. katsumadai is due to its antioxidant (Lee et al., 2003) and anti-inflammatory activities (Noh, 1998; Choi et al., 2009). The seeds contain eucalyptol, a-humulene, trans,trans-farnesol(I), linalool, camphor, terpinen-4-ol, carvotanacetone, bornyl acetate, geranyl acetate, methyl cinnamate, nerolidol, alpinetin, cardamomin and diarylheptanoids (Yushiro et al., 1968; Saiki et al., 1978; Kuroyanagi et al., 1983; Brown and Rice-Evans, 1998) and it has been reported that eucalyptol and terpinen-4-ol have antinociceptive activity (Santos and Rao, 2000; Moreira et al., 2001).

It has been reported that flavonoids and chalcone possess antinociceptive activity (Kekesi et al., 2003; de Campos-Buzzi et al., 2006); therefore, these compounds in $A$. katsumadai may contribute to its antinociceptive activity. In addition, cardamonin, one of the major active constituents of $A$. katsumadai, was found to inhibit COX-2 and iNOS expression via inhibition of the NF-кB pathway in RAW 264.7 macrophage cells (Lee et al., 2006); therefore, this compound may be involved in the antinociceptive activity of $A$. katsumadai.

In conclusion, the results of this study demonstrated that the mechanism of the antinociceptive activity of $A$. katsumadai is not mediated by opioid receptors, but instead via suppression of COX-2, which differs from $A$. zerumbet and $A$. calcarata. Further studies are ongoing to elucidate the components of $A$. katsumadai responsible for its antinociceptive activity.

\section{ACKNOWLEDGMENTS}

This work was supported by the Research Program for New Drug Target Discovery through the National Research Foundation of Korea (NRF) funded by the Ministry of Education, Science and Technology (2009-0083364).

\section{REFERENCES}

Arambewela, L. S., Arawwawala, L. D. and Ratnasooriya, W. D. (2004). Antinociceptive activities of aqueous and ethanolic extracts of Alpinia calcarata rhizomes in rats. J. Ethnopharmacol. 95, 311-316.

Bjorkman, R., Hallman, K. M., Hedner, J., Hedner, T. and Henning, M. (1994). Acetaminophen blocks spinal hyperalgesia induced by NMDA and substance P. Pain 57, 259-264.

Brown, J. E. and Rice-Evans, C. A. (1998). Luteolin-rich artichoke extract protects low density lipoprotein from oxidation in vitro. Free Radic. Res. 29, 247-255.

Calixto, J. B., Campos, M. M., Otuki, M. F. and Santos, A. R. (2004). Anti-inflammatory compounds of plant origin. Part II. modulation of pro-inflammatory cytokines, chemokines and adhesion molecules. Planta Med. 70, 93-103.

Ching, S. (1978). The encyclopedia of Chinese materia medica. Shanghai Science \& Technical Publishers, Shanghai.

Choi, J. K., Kim, K. M., Kim, D. K., Yeom, M. H., Koh, J. Y., Jung, S. J., Kim, H. J., Oh, S. H., Kim, S. Y. and Lee, C. H. (2009). Topical anti-inflammatory and antipruritic effects of Alpinia katsumadai extracts. J. Dermatol. Sci. 53, 81-84.

de Araujo, P. F., Coelho-de-Souza, A. N., Morais, S. M., Ferreira, S. C. and Leal-Cardoso, J. H. (2005). Antinociceptive effects of the essential oil of Alpinia zerumbet on mice. Phytomedicine 12, 482-486.

de Campos-Buzzi, F., Pereira de Campos, J., Pozza Tonini, P., Correa, R., Augusto Yunes, R., Boeck, P. and CechinelFilho, V. (2006). Antinociceptive effects of synthetic chalcones obtained from xanthoxyline. Arch. Pharm. (Weinheim) 339, 361-365.

Dong, H., Mei, Q., Xu, G. and Xu, L. (1992). Textual study on traditional Chinese drugs caodoukou and baidoukou. Zhongguo Zhong Yao Za Zhi 17, 451-453, 509.

Dunham, N. W. and Miya, T. S. (1957). A note on a simple apparatus for detecting neurological deficit in rats and mice. J. Am. Pharm. Assoc. Am. Pharm. Assoc. (Baltim) 46, 208209.

Gracioso, J. S., Paulo, M. Q., Hiruma Lima, C. A. and Souza Brito, A. R. (1998). Antinociceptive effect in mice of a hydroalcoholic extract of Neurolaena lobata (L.) R. Br. and its organic fractions. J. Pharm. Pharmacol. 50, 1425-1429.

Hendershot, L. C. and Forsaith, J. (1959). Antagonism of the frequency of phenylquinone-induced writhing in the mouse by weak analgesics and nonanalgesics. J. Pharmacol. Exp. Ther. 125, 237-240.

Kekesi, G., Dobos, I., Benedek, G. and Horvath, G. (2003). Antinociceptive activity of Sempervivum tectorum L. extract in rats. Phytother. Res. 17, 1032-1036.

Kim, J. H., Lee, E. J., Shin, D. O., Hong, S. E. and Kim, J. K. (2000). Protective effect against oxidative stress in medicinal plant extract. J. Korean Assoc. Rad. Protec. 25, 37-43.

Kuroyanagi, M., Noro, T., Fukushima, S., Aiyama, R., lkuta, A., Itokawa, H. and Morita M. (1983). Studies on the constituents of the Seeds of Alpinia katsumadai. Chem. Pharm. Bull. 31, 1544-1550.

Lee, J. H., Jung, H. S., Giang, P. M., Jin, X., Lee, S., Son, P. T., Lee, D., Hong, Y. S., Lee, K. and Lee, J. J. (2006). Blockade of nuclear factor-kappaB signaling pathway and anti-inflammatory activity of cardamomin, a chalcone analog from Alpinia conchigera. J. Pharmacol. Exp. Ther. 316, 271-278.

Lee, S. E., Shin, H. T., Hwang, H. J. and Kim, J. H. (2003). Antioxidant activity of extracts from Alpinia katsumadai seed. 
Phytother. Res. 17, 1041-1047.

Mendell, J. R. and Sahenk, Z. (2003). Clinical practice. Painful sensory neuropathy. N. Engl. J. Med. 348, 1243-1255.

Moreira, M. R., Cruz, G. M., Lopes, M. S., Albuquerque, A. A. and Leal-Cardoso, J. H. (2001). Effects of terpineol on the compound action potential of the rat sciatic nerve. Braz. J. Med. Biol. Res. 34, 1337-1340.

Noh, M. S., Ha, J. Y., Lee, C. H., Lee, W. Y., Lee, S. H. and Lee J. J. (1998). Inhibitory activities of natural products on lipopolysaccharide induced prostaglandin production in mouse macrophages. Yakhakhoe Chi 42, 558-566.

Ouellet, M., Riendeau, D. and Percival, M. D. (2001). A high level of cyclooxygenase-2 inhibitor selectivity is associated with a reduced interference of platelet cyclooxygenase-1 inactivation by aspirin. Proc. Natl. Acad. Sci. U. S. A. 98 14583-14588.

Randall, L. O. and Selitto, J. J. (1957). A method for measurement of analgesic activity on inflamed tissue. Arch. Int. Pharmacodyn. Ther. 111, 409-419.

Rang, H. P., Dale, M. M. and Ritter, J. M. (1978). Pharmacology. Churchill Livingstone, London.

Saiki, Y., Ishikawa, Y., Uchida, M. and Fukushima, S. (1978). Essential oil from chinese drug 'caodoukou', the seeds of Alpinia katsumadai. Phytochemistry 17, 808-809.

Santos, F. A. and Rao, V. S. (2000). Antiinflammatory and antinociceptive effects of 1,8-cineole a terpenoid oxide present in many plant essential oils. Phytother. Res. 14, 240-244.

Siegmund, E., Cadmus, R. and Lu, G. (1957). A method for evaluating both non-narcotic and narcotic analgesics. Proc. Soc. Exp. Biol. Med. 95, 729-731.

Smith, C. J., Zhang, Y., Koboldt, C. M., Muhammad, J., Zweifel B. S., Shaffer, A., Talley, J. J., Masferrer, J. L., Seibert, K. and Isakson, P. C. (1998). Pharmacological analysis of cyclooxygenase-1 in inflammation. Proc. Natl. Acad. Sci. U. S. A 95, 13313-13318

Steen, K. H., Steen, A. E., Kreysel, H. W. and Reeh, P. W. (1996). Inflammatory mediators potentiate pain induced by experimental tissue acidosis. Pain 66, 163-170.

Winter, C. A., Risley, E. A. and Nuss, G. W. (1962). Carrageenin-induced edema in hind paw of the rat as an assay for antiiflammatory drugs. Proc. Soc. Exp. Biol. Med. 111, 544547.

Yang, Y., Kinoshita, K., Koyama, K., Takahashi, K., Tai, T. Nunoura, Y. and Watanabe, K. (1999). Two novel antiemetic principles of Alpinia katsumadai. J. Nat. Prod. 62, 1672-1674.

Yushiro, K., Shuhiti, T. and Ikuo, Y. (1968). Studies on the constituents of the seeds of Alpinia katsumadai. Yakugaku Zasshi 88, 239-241. 